\title{
Rare Intramedullary Myeloid Sarcoma Mistaken as Acute Transverse Myelitis
}

\author{
Min-Gi Lee, Jung-Woo Hur, Stephen Ahn \\ Department of Neurosurgery, Seoul St. Mary's Hospital, College of Medicine, The Catholic University of Korea, Seoul, \\ Republic of Korea
}

Corresponding author: Jung-Woo Hur

Department of Neurosurgery,

Seoul St. Mary's Hospital,

College of Medicine, The

Catholic University of Korea,

222 Banpo-daero, Seocho-gu,

Seoul 06591, Republic of Korea

Tel: +82-2-2258-6353

Fax: +82-2-594-4248

E-mail: neurotique79@gmail.com

Received: August 21, 2018

Revised: August 30, 2018

Accepted: September 1, 2018
Myeloid sarcoma is a rare extramedullary tumor of immature myeloid cells. It more often develops with the underlying acute myeloid leukemia, but it can also occur with chronic myeloid leukemia $(\mathrm{CML})$, myelodysplastic syndrome and rarely even with the absence of marrow involvement. Spinal epidural myeloid sarcoma is uncommon, and intramedullary presentation is exceptionally rare. In this report the authors report an unusual case of spinal intramedullary myeloid sarcoma after completer remission from CML several years ago. He was mistakenly diagnosed as acute transverse myelitis and treated with high-dose steroid and plasmaparesis without improvement. Magnetic resonance imaging revealed intramedullary mass-like lesion with hyper-intensity in T2-weighted image and mild homogeneous enhancement. Myeloid sarcoma should be considered as a possible differential diagnosis when patient with history of myeloid leukemia present intramedullary tumor and pathologic confirmation with open biopsy is mandatory for the exact diagnosis.

Key Words: Leukemia, myelogenous, chronic, BCR-ABL positive; Myelitis, transverse; Sarcoma, myeloid

\section{INTRODUCTION}

Myeloid sarcoma or granulocytic sarcoma is an unusual tumor of immature cells of the myeloid origin ${ }^{8}$. The tumor is reported in $0.7 \%$ to $9.0 \%$ of patients with acute or chronic myeloid leukemia (CML) and usually is seen either at the time of initial presentation or during some phase of the disease ${ }^{7}$. However, in some rare cases, it may present as recurrence of leukemia before it becomes apparent clinically". Common sites of occurrence are skin, soft tissue, bone, periosteum and lymph nodes, with rare involvement of central nervous system ${ }^{1,12)}$. In spinal involvement, myeloid sarcoma usually occurs as an epidural mass with signs of cord compression ${ }^{6,716)}$. However, a few cases of rare intramedullary involvement have been reported worldwidely ${ }^{2,9,15}$. We report an extremely rare case of intramedullary myeloid sarcoma in a patient with CML as a hematologic relapse.

\section{CASE REPORT}

A 58-year-old male was transferred to neurology division under the diagnosis of suspicious acute transverse myelitis. $\mathrm{He}$ suffered from progressive paraparesis and voiding difficulty for several months. The patient had a medical history of CML and underwent allogenic bone marrow transplantation 13 years ago. He had complete remission from the disease and had no neurological deterioration.

The patient developed sudden both lower extremities weakness and gait disturbance without any trauma history. He was treated with conservative treatment in local clinic for several month but the symptom was aggravated with newly developed urinary and defecation difficulties. He was transferred to tertiary medical center for the further treatment. Initial magnetic resonance imaging (MRI) with gadolinium enhancement revealed
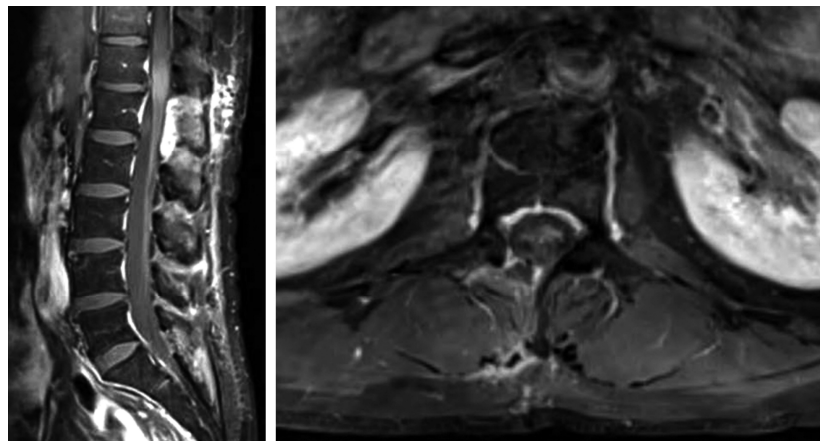

Fig. 1. Initial magnetic resonance imaging with gadolinium enhancement revealed an about $3 \mathrm{~cm}$ sized intramedullary lesion with slight T2 hyper-intensity and mild homogeneous enhancement at T12-L1 level. 


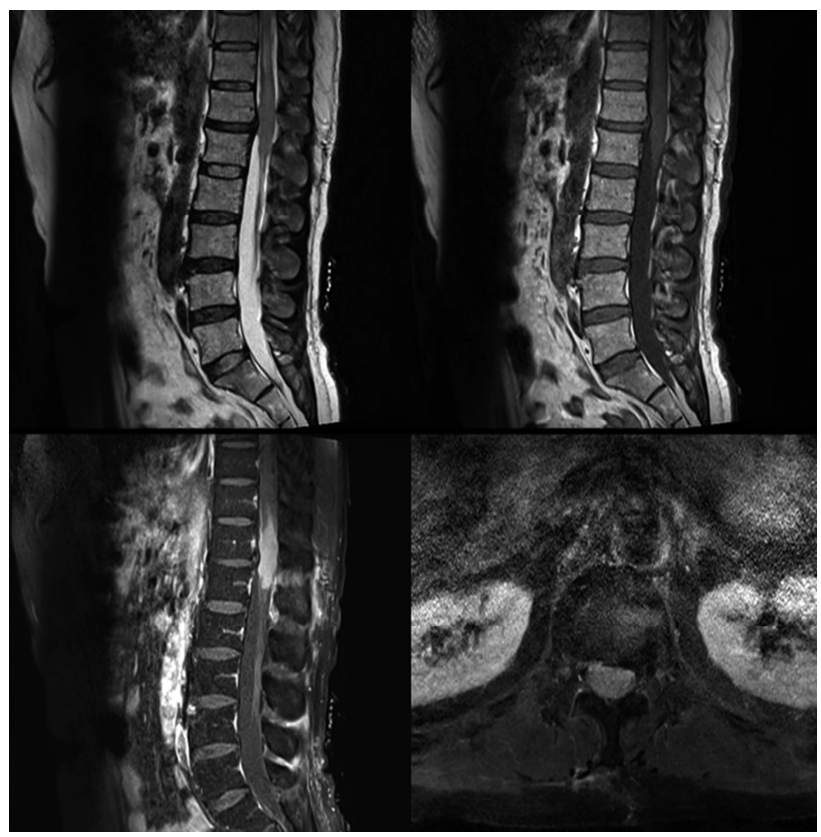

Fig. 2. Enhanced spinal magnetic resonance imaging after the admission showed increase in size of intramedullary enhancing mass lesion with newly noted syrinx and postoperative change at T12-L1 level.

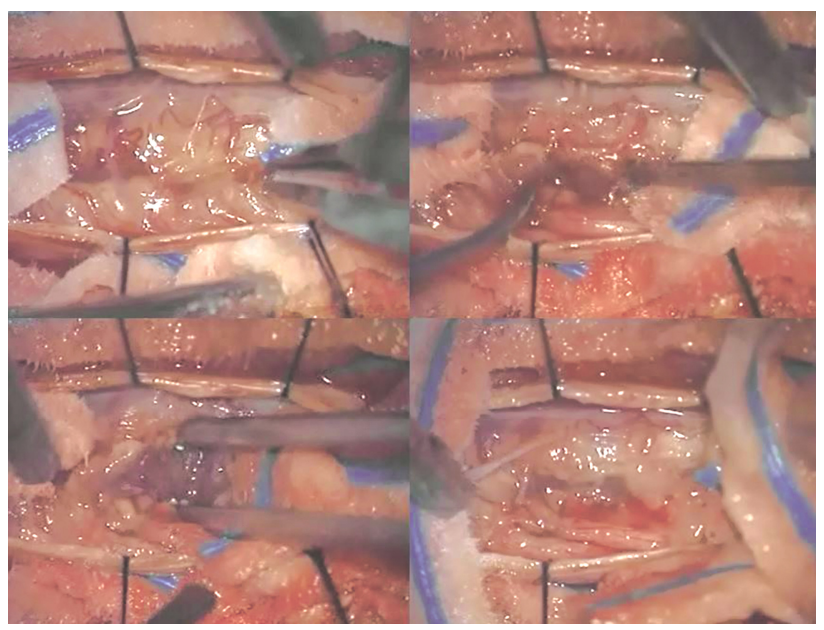

Fig. 3. Extended T12 laminectomy and mid-line myelotomy with open biopsy was done under general anesthesia.

an about $3 \mathrm{~cm}$ sized intramedullary lesion with slight $\mathrm{T} 2$ hyperintensity and mild homogeneous enhancement at T12-L1 level (Fig. 1). Open biopsy with cerebrospinal fluid cytology study was negative for malignancy, hematologic disorders and infection. His final diagnosis was acute transverse myelitis and was treated with high-dose steroid injection and plasmaparesis for 7 days without any neurological improvement. He was then transferred to rehabilitation center for 2 month of comprehensive rehabilitation and physical therapy. His neurological symptom worsened gradually against all efforts and was consulted to our institution for the second opinion.

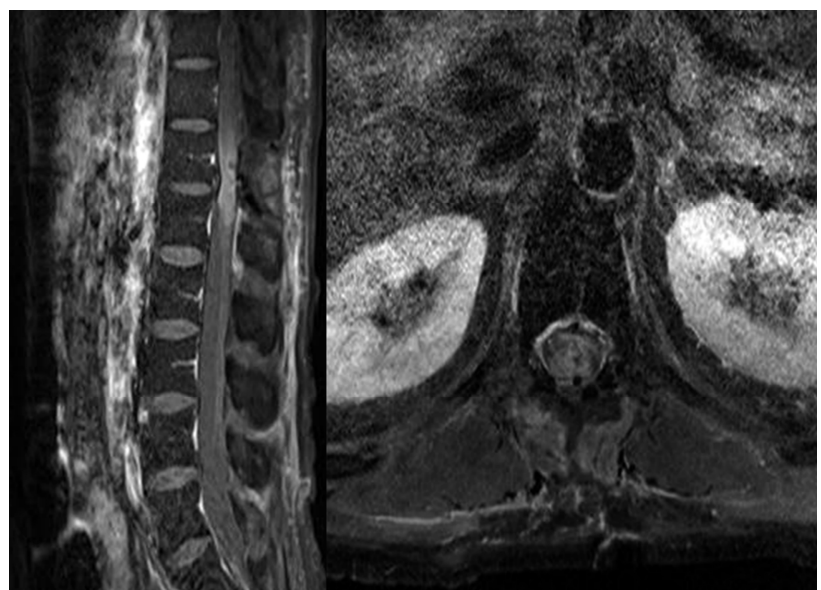

Fig. 4. Postoperative magnetic resonance imaging showed no immediate complication related to the operations.

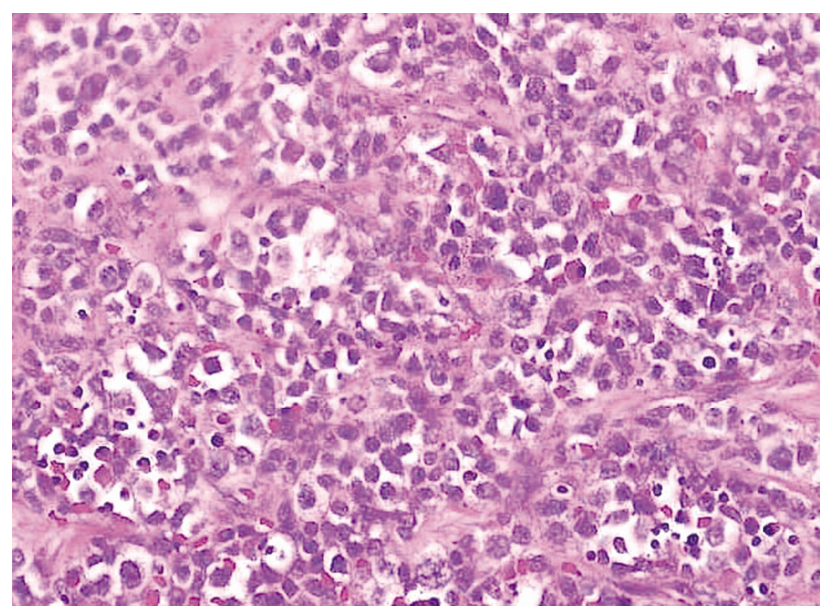

Fig. 5. Pathologic findings suggest soft tissue mass with myeloid leukemic blasts infiltration suggesting myeloid sarcoma. Histologic findings suggested presence of numerous blasts with round or oval nucleus, dispersed chromatin and prominent nucleolus. The cytoplasm of the majority of neoplastic cells was agranular with a varying degree of basophilia. Eosinophilic granulation was notable in a minority of a cell population.

Initial neurological examination demonstrated grade I motor weakness of both lower extremities and severe hypesthesia below L1 dermatome without any long tract signs. Laboratory examination revealed mild lymphocytosis (52.8\%) without any sign of hematologic disorder or infection. Enhanced spinal MRI after the admission showed increase in size of intramedullary enhancing mass lesion with newly noted syrinx and postoperative change at T12-L1 level (Fig. 2). Our initial impression was granulocytic sarcoma or atypical infectious myelitis.

Patient was consulted to the neurosurgery department for the open biopsy. Extended T12 laminectomy and mid-line myelotomy with open biopsy was done under general anesthesia (Fig. 3). Frozen biopsy revealed malignancy with atypical small 
round cells. There was no complication related to the operations (Fig. 4). Final pathologic confirmation was myeloid sarcoma with $80 \%$ Ki-67 and positive CD117 in immunohistochemistry. Histologic findings suggested presence of numerous blasts with round or oval nucleus, dispersed chromatin and prominent nucleolus. The cytoplasm of the majority of neoplastic cells was agranular with a varying degree of basophilia. Eosinophilic granulation was notable in a minority of a cell population (Fig. 5). After histologic confirmation the patient was transferred to hematologic department for the further treatment.

\section{DISCUSSION}

Myeloid sarcoma was first described in 1,811 and represents a rare hematologic phenomenon which occurs in patients with myeloid leukemia ${ }^{3,12)}$. The prevalence of myeloid sarcoma in the spine is estimated to be less than $1 \%$ among all patients with acute and CML. All levels of the spine can be affected, but more frequently in the lumbosacral spine, followed by the thoracic spine and cervical level ${ }^{7,15-17)}$. The clinical manifestation of spinal myeloid sarcoma varies, and it largely depends on the tumor location and size ${ }^{4,5,9,14)}$. Most spinal myeloid sarcoma that arises in the epidural or paraspinal tissues can present with signs of cord compression due to mass effect ${ }^{2,9,11}$. In the presented case, the patient's initial presentation was paraparesis due to unusual intramedullary location. This unspecific presentation could easily be misdiagnosed with atypical inflammatory condition such as acute transverse myelitis and demyelinating diseases, which could lead to prolonged and inappropriate treatment and delay the timely diagnosis $5,7,12,16$ ).

The diagnosis of spinal myeloid sarcoma is often challenging because the clinical and laboratory findings are usually not contributory ${ }^{5,10)}$. The radiologic findings are usually non-specific, and the mass often appears as a soft tissue mass ${ }^{2}$. On MRI examination, spinal myeloid sarcoma yields a similar signal intensity to muscle on T1-weighted images (WIs) and intermediate signal intensity on T2-WIs with subtle to intermediate enhancement with Gadolinium ${ }^{1,4,14)}$. Immunohistochemistry is the most accurate method for establishing the diagnosis of myeloid sarcoma, and a series of markers can be used for diagnosis confirmation and further lineage characterization ${ }^{6,9,15,16)}$.

Myeloid sarcoma can rarely occur in patients without any evidence of leukemia and may precede the development of systemic disease ${ }^{5,9)}$. If the patient has established leukemia, the diagnosis of myeloid sarcoma is relatively easy and it is suggested to always be included in the differential diagnosis for patients with leukemia with a soft tissue mass ${ }^{6,13)}$. The diagnosis of myeloid sarcoma could be difficult if there is no clinical history of leukemia and every effort should be made to obtain a pathologic diagnosis ${ }^{4,7,9)}$. In the present case, the patient underwent repeated open biopsy and immunohistochemical stains showed positive staining in the atypical, which helped to confirm the diagnosis.
The treatment strategy for myeloid sarcoma largely depends on systemic manifestation of leukemic cells and whether it is initial diagnosis or relapse ${ }^{6,15-17)}$. The optimal timing and treatment of isolated myeloid sarcoma is still controversial, however, delayed or inadequate systemic treatment will almost always progress to systemic infiltrations ${ }^{6,8,10,14)}$. For the patient with myeloid sarcoma who presents with concurrent marrow involvement, systemic treatment is always suggested ${ }^{5,9,16)}$. Overall, prompt diagnosis and adequate systemic treatment are essential for a good outcome.

\section{CONCLUSION}

We report a rare case of spinal intramedullary myeloid sarcoma presenting as relapse of CML. Diagnosis may be difficult and knowledge of its clinical presentation, imaging, and histologic characterization can lead to proper diagnosis and appropriate treatment. Although rare, myeloid sarcoma should be prominent in the differential diagnosis whenever a history of leukemia is present in a patient presenting with myelopathy symptoms and unusual intramedullary mass-like lesion.

\section{CONFLICT OF INTEREST}

No potential conflict of interest relevant to this article was reported.

\section{REFERENCES}

1. Best-Aguilera CR, Vazquez-Del Mercado M, Muñoz-Valle JF, Herrera-Zarate L, Navarro-Hernandez RE, Martin-Marquez BT, et al.: Massive myeloid sarcoma affecting the central nervous system, mediastinum, retroperitoneum, liver, and rectum associated with acute myeloblastic leukaemia: a case report. J Clin Pathol 58:325-327, 2005

2. Cho SF, Liu TC, Chang CS: Isolated central nervous system relapse presenting as myeloid sarcoma of acute myeloid leukemia after allogeneic peripheral blood stem cell transplantation. Ann Hematol 92:133-135, 2013

3. Devi VL, Doval DC, Rao CR, Bapsy PP: Spinal epidural granulocytic sarcoma: A rare presentation of acute myeloid leukemia. Ann Saudi Med 16:212-214, 1996

4. Eom KS, Kim TY: Intraparenchymal myeloid sarcoma and subsequent spinal myeloid sarcoma for acute myeloblastic leukemia. J Korean Neurosurg Soc 49:171-174, 2011

5. Hu X, Shahab I, Lieberman IH: Spinal myeloid sarcoma "Chloroma" presenting as cervical radiculopathy: Case report. Global Spine J 5:241-246, 2015

6. Isshiki Y, Ohwada C, Togasaki E, Shimizu R, Hasegawa N, Yamazaki A, et al.: Acute myeloid leukemia concurrent with spinal epidural extramedullary myeloid sarcoma accompanied by a high CD25 expression and the FLT3-ITD mutation. Intern Med 53:1159-1164, 2014 
7. Kyaw TZ, Maniam JA, Bee PC, Chin EF, Nadarajan VS, Shanmugam $\mathrm{H}$, et al.: Myeloid sarcoma: an unusual presentation of acute promyelocytic leukemia causing spinal cord compression. Turk J Haematol 29:278-282, 2012

8. Landis DM, Aboulafia DM: Granulocytic sarcoma: an unusual complication of aleukemic myeloid leukemia causing spinal cord compression. A case report and literature review. Leuk Lymphoma 44:1753-1760, 2003

9. Olar A, Lapadat R, Davidson CJ, Stein TD, Dahiya S, Perry A, et al.: Central nervous system involvement by myeloid sarcoma: a report of 12 cases and review of the literature. Clin Neuropathol 35:314-325, 2016

10. Serefhanoglu S, Goker H, Aksu S, Buyukasik Y, Sayinalp N, Haznedaroglu IC, et al.: Spinal myeloid sarcoma in two nonleukemic patients. Intern Med 49:2493-2497, 2010

11. Stepensky P, Revel-Vilk S, Yehuda-Gafni O, Mali B, Resnick IB, Weintraub M: Isolated central nervous system granulocytic sarcoma and meningeal myeloid leukemia: successful treatment without radiotherapy. Isr Med Assoc J 11:569-570, 2009

12. Struhal W, Oberndorfer S, Lahrmann H, Lindeck-Pozza E, Hess
B, Nussgruber V, et al.: Myeloid sarcoma in the central nervous system: case report and review of the literature. Acta Clin Croat 47:19-24, 2008

13. Takeda M, Yamaguchi S, Eguchi K, Kajiume T, Nishimura S, Kobayashi M, et al.: Spinal epidural granulocytic sarcoma in a child precedent to clinical manifestation of acute myeloid lymphoma: case report. Neurol Med Chir (Tokyo) 49:221-224, 2009

14. Widhalm G, Dietrich W, Müllauer L, Streubel B, Rabitsch W, Kotter MR, et al.: Myeloid sarcoma with multiple lesions of the central nervous system in a patient without leukemia. Case report. J Neurosurg 105:916-919, 2006

15. Yang B, Yang C, Fang J, Yang J, Xu Y: Clinicoradiological characteristics, management and prognosis of primary myeloid sarcoma of the central nervous system: A report of four cases. Oncol Lett 14:3825-3831, 2017

16. Yang C, Fang J, Xu Y: Isolated spinal myeloid sarcoma with rapid progression. Spine J 16:e517-e518, 2016

17. Yang C, Liu Y, Li G, Bai J, Qian J, Xu Y: Multifocal myeloid sarcoma in the central nervous system without leukemia. Clin Neurol Neurosurg 120:99-102, 2014 